\title{
Face Recognition using Template Matching
}

\author{
Amandeep Kour \\ Assistant Professor \\ Dept. of Computer Sciences and Engineering, \\ "M.B.S.C.E.T",Babliana, JAMMU \\ Jammu and Kashmir
}

\begin{abstract}
The traditional approach towards human identification such as fingerprints, identity cards, iris recognition etc. lead to the improvised technique for face recognition. This includes enhancement and segmentation of face image, detection of face boundary and facial features, matching of extracted features against the features in a database, and finally recognition of the face. This research proposes a wavelet transformation for preprocessing the face image, extracting edge image, extracting features and finally matching extracted facial features for face recognition. Simulation is done using ORL database that contains PGM images. This research finds application in homeland security where it can increase the robustness of the existing face recognition algorithms.
\end{abstract}

\section{Keywords}

Face recognition, Wavelet transformation, edge image extraction, feature extraction, match features

\section{INTRODUCTION}

THE human face plays an important role in our Social interaction, conveying people's identity. Using human face as a key for security, the biometrics face recognition technology has received significant attention in the past several years due to its potential for a wide variety of applications in both law enforcement and non-law enforcement. The face recognition technology has become important because of its proven efficiency for enhanced security without notifying the person. An automated face recognition system is useful for identification and recognition of humans that may find application in criminal identification, surveillance systems, video telephony, and credit card verification. As compared with other biometrics systems using finger print/palm print and iris, face recognition has distinct advantages because of its non-contact process. Face images can be captured from a distance without touching the person being identified, and the identification does not require interacting with the person. Most of the face recognition methods are knowledge -based, feature-based, image-based.

The template matching is simple technique for image processing topics like feature extraction, edge detection, object extraction. Template matching can be subdivided between two approaches: feature-based and template-based matching. The feature-based approach uses the features of the search and template image, such as edges or corners, as the primary match-measuring metrics to find the best matching location of the template in the source image. The template-based, or global, approach uses the entire template. To recognize a human face, some special features need to be extracted. These special features include eyes, nose, mouth, and chin along with the shape of the face. To locate these features, researchers have proposed a variety of methods based on symmetry of faces [1], facial geometry and luminance [2], and template matching [3]. Generally, the vision based face recognition can be explained as follows. Initially, the subject image is enhanced and segmented. Then the contour features of the face are extracted by contour extraction method, and compares with the extracted features of the database image [4]. If there is a match then the person in the subject image is recognized.

\section{PROPOSED APPROACH}

The proposed approach employs wavelet based decomposition technique

\subsection{Wavelet Decomposition}

The continuous wavelet transform of a 1-D signal $f(x)$ is defined as

$\left(W_{a} f\right)(b)=\int f(x) \psi \quad a, b(x) d x$

where the wavelet a,b $\Psi$ is computed from the mother wavelet $\Psi$ by translation and dilation[5],

$$
\psi_{a, b(x)=} \frac{1}{\overline{|a|} \psi((x-a) / b)}
$$

under some mild assumptions, the mother wavelet $\Psi$. Satisfies the constraint of having zero mean [7]. The eq. (1) can be discretized by restraining $a$ and $b$ to a Discrete lattice $(a=2 b, b \in l)$. Typically it is imposed that the transform should be non-redundant, complete and constitutes a multiresolution representation of the original signal. The extension to the $2-\mathrm{D}$ cases is usually performed by using a product of $1-\mathrm{D}$ filters. In practice, the transform is computed by applying a separable filter bank to the image

$$
\begin{aligned}
\text { An } & =[\mathrm{Hx} *[\mathrm{Hy} * \mathrm{An} 1] \downarrow 2,1] \downarrow 1,2 \\
\text { Dn } 1 & =[\mathrm{Hx} *[\mathrm{~Gy} * \mathrm{An} 1] \downarrow 2,1] \\
\text { Dn2 } & =[\mathrm{Gx} *[\mathrm{Hy} * \mathrm{An} 1] \downarrow 2,1] \\
\text { Dn3 } & =[\mathrm{Gx} *[\mathrm{~Gy} * \mathrm{An} 1] \downarrow 2,1] \downarrow 1,2
\end{aligned}
$$

\section{Figures}

Where $*$ denotes the convolution operator, $\downarrow 2,1(\downarrow 1,2)$ denotes the down sampling along the rows (columns) and $\mathrm{A}_{0}$ $=\mathrm{I}$ is the original image, $\mathrm{H}$ and $\mathrm{G}$ are low pass and high pass filters, respectively. The term $A_{n}$ is obtained by low pass filtering and is referred to as the low resolution (Approximation) image at scale $\mathrm{n}$. The terms $\mathrm{D}_{\mathrm{n} 1}, \mathrm{D}_{\mathrm{n} 2}, \mathrm{D}_{\mathrm{n} 3}$ are obtained by band pass filtering in a specific direction (Horizontal, Vertical and Diagonal, respectively) and thus contain directional detail information and are referred to as 
high resolution (Detail) images at scale $\mathrm{n}$. The original image $\mathrm{I}$ is thus represented by a set of sub images at several scales. This decomposition is called

"Pyramidal wavelet transform" decomposition or discrete wavelet decomposition (DWT). Every detail sub image contains information of a specific scale and orientation. The spatial information is retained within the sub image. In the present paper, the features are obtained using Haar Wavelet (Fig. 1.), which is given by

$\left\{\begin{array}{ccc} & 1 & 0 \leq t \leq 12 \\ \psi & (t) & \\ \mid & 0 & \end{array}\right.$

(4)

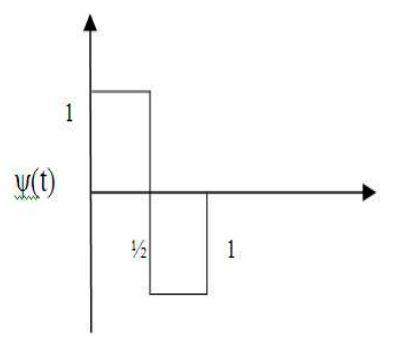

Fig.1. Haar Wavelet representation
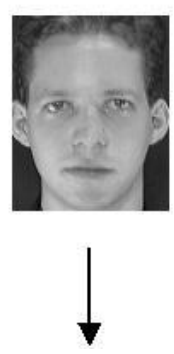

A1 D11

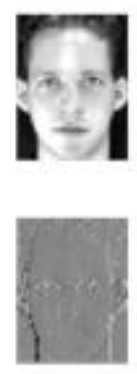

D12
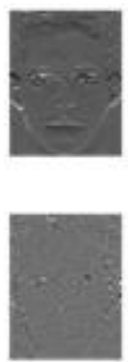

D13
Fig.2 Wavelet Decomposition of an Image

As is required, $\psi(\mathrm{t})$ integrates to zero. One-level decomposition is performed on image I, which yields approximation image (A1) and horizontal, vertical and diagonal detail images (D11, D12, D13) [6]as shown in Fig. 2

\subsection{Edge Image Extraction}

The wave decomposed sub bands are further used forming the edge images. The strong edge responses from the horizontal, vertical and diagonal components are made use of for this purpose [7]. The algorithm for achieving this is presented in figure 2.2 below:

\section{ALGORITHM:}

Step1. Resize the H (Horizontal), V (vertical) and D (diagonal) components to the original size of the image.

Step 2. $\operatorname{Img} 1=$ mat2gray $(\mathrm{H}+\mathrm{V}+\mathrm{D})$, where mat2gray maps the responses to the gray range $(0-255)$.

Step 3. Img2=mat2gray $((\operatorname{abs}(\mathrm{H})+\operatorname{abs}(\mathrm{V})+\operatorname{abs}(\mathrm{D})))($ The H, $\mathrm{V}$ and $\mathrm{D}$ components contain both positive and negative responses, convert all the responses as positive. The stronger edges are the high order responses in these subbands).

Step4. $\quad$ Img3 $=$ mat2gray $\quad(($ mat2gray $(H)+\quad$ mat2gray $(\mathrm{V})$ +mat2gray(D)))

Step 5. Img4 = mat2gray (abs (Img1 - Img3)) Step 6. Return Img1, Img2, Img3, Img4.

The sample face images are shown in Fig

\section{DWT}

Img 1
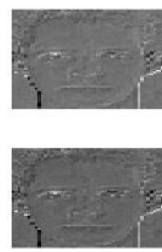

Img 3
Img 2

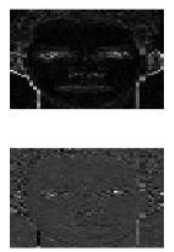

Img4

\subsection{Feature Extraction}

These edge images are used as face templates and shape estimates of these images is carried out next. For shape estimation we employ moment invariants [8]. The moment invariants are given by:

Moments of Two-Dimensional Functions

For a 2-D continuous function $\mathrm{f}(\mathrm{x}, \mathrm{y})$, the moment of order $(p+q)$ is defined as

$$
\begin{gathered}
\infty \propto \\
p \quad q \\
{ }^{m} p q=\int_{\int}^{x} y \quad f(x, y) d x d y
\end{gathered}
$$

For $\mathrm{p}, \mathrm{q}=0,1,2 \ldots$ A uniqueness theorem states that if $\mathrm{f}(\mathrm{x}, \mathrm{y})$ is piecewise continuous and nonzero values only in finite part of the xy-plane, moments of all the order exist, and the moment sequence $\left(m_{p q}\right)$ uniquely determines $f(x, y)$.

The central moments are defined as

$$
\begin{array}{cc}
\infty \quad \infty \quad-{ }_{p} \quad-q \\
\mu_{p q=} \quad f(x-x) \quad(y-y) f(x, y) d x d y \\
\text { Where } \\
\bar{x}=\frac{m_{10}}{m_{00}} \quad \text { and } \bar{y}=\frac{m_{01}}{m_{00}}
\end{array}
$$


If $f\left(x_{0} x\right)$ is a digital image, then (6) becomes $\mu_{s x<}=\sum \sum_{*}(x-x)^{-p}(y-y) f(x, y)$

The central moments of order up to 3 are

$$
2000=\sum \sum(x-x)(y-y) j f(x, y)
$$$$
=\sum_{x} \sum_{x} f(x, y)
$$$$
=m a
$$$$
\text { 서을 }=\sum \sum(x-x)^{1}\left(y^{-} y\right)^{0} f(x, y)
$$$$
=m_{10}-m_{10} / \sim_{-0}\left(m_{00}\right)
$$$$
=0
$$$$
\mu_{01}=\sum \sum(x-\bar{x})^{0}(y-y)^{1} f(x, y)
$$$$
=m_{01}-m_{01} / m_{c o}\left(m_{00}\right)
$$$$
=0
$$$$
\mu_{11}=\sum_{z} \sum_{z}(x-x)^{1}(y-y)^{1} f(x, y)
$$$$
=m_{1:}-m_{01} m 00\left(m_{10}\right)
$$$$
=m_{11}-x m_{01}=m_{11}-y m_{10}
$$$$
\angle C O=\sum_{z} \sum_{y}(x-x)^{2}(y-y)^{1} f(x, y)
$$$$
=m_{10}-\frac{2 m^{2}}{m_{10}^{10}}+\frac{m^{2}}{m_{10}^{10}}
$$$$
=m_{10}-\frac{m^{2}}{m_{00}}
$$$$
=m_{20}-\bar{x} m_{10}
$$$$
\mu_{02}=\sum_{\overline{5}} \sum(x-\bar{x})^{0}(y-y)^{2} f(x, y)
$$$$
=m_{02}-\frac{m^{2}}{m_{\omega 0}}
$$$$
=m_{02}-\bar{y} m_{10}
$$$$
\mu_{11}=\sum_{x} \sum_{\nu}(x-\bar{x})^{1}(y=y)^{1} f(x, y)
$$$$
=m_{11}-\frac{m_{10} m_{01}}{m_{00}}
$$$$
=m_{11}-x_{01}=m_{11}-m_{2} m_{10}
$$$$
\text { LEO }=\sum \sum(x-x)(y-y) \quad f(x, y)
$$$$
=m_{20}-\frac{2 m^{2} 10}{m_{\omega 0}}+\frac{m^{2}}{m}
$$$$
=m_{20}-\frac{m^{2}}{m_{00}}
$$$$
=m_{20}-\bar{x} m_{10}
$$$$
\mu_{02}=\sum_{\xi} \sum_{y}(x-x)^{0}(y-y)^{2} f(x, y)
$$$$
=m_{n}-\frac{m_{n}^{2}}{m_{w 0}}
$$$$
=m_{02}-\bar{y} m_{01}
$$$$
\mu_{21}=\sum_{z} \sum_{y}(x-\bar{x})^{2}(y-y)^{1} f(x, y)
$$$$
=m_{21}-\overline{2 x} m_{11}-\bar{y}_{20}+\overline{2 x}^{2} m_{01}
$$$$
\mu_{12}=\sum_{z} \sum_{\gamma}(x-x)^{1}(y-\bar{y})^{2} f(x, y)
$$$$
=m_{12}-2 \bar{y} m_{11}-\bar{x} m_{02}+2 \bar{y}_{m_{10}}
$$$$
\mu_{50}=\sum_{z} \sum_{y}(x-\bar{x})^{3}(y-y)^{0} f(x, y)
$$$$
=m_{30}-\overline{3} x m_{20}+\overline{2} x^{2} m_{01}
$$

$$
\begin{aligned}
\mu_{03}= & \sum \sum(x-x)^{0}(y-y)^{3} f(x, y) \\
& - \\
= & m_{03}-\overline{3 y} m_{02}+2 y^{2} m_{01}
\end{aligned}
$$

(8)

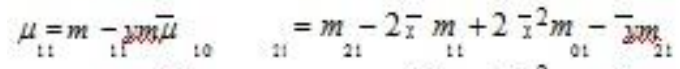

$$
\begin{aligned}
& \mu_{20} \quad 20 \quad-\frac{m}{10}-x_{12} \mu-m-\overline{2}_{11} y_{11}+2 \bar{y}^{2} m_{10}-\bar{x}_{02}
\end{aligned}
$$

$$
\eta_{\infty}=\frac{\mu_{\infty}}{\mu_{0}^{\gamma}}
$$

Where

$$
\begin{aligned}
& \gamma=\frac{p+q}{2}+1 \\
& \text { For } \mathrm{p}+\mathrm{g}=2,3
\end{aligned}
$$

A set of seven invariant moments can be derived from the second and third moments.

$$
\begin{aligned}
& \phi_{1}=\eta_{20}+\eta_{02} \\
& \phi=\left(\eta_{2}-\eta_{02}{ }^{2}+4 r_{1:}^{2}\right. \\
& \phi=\left(\eta-3 \eta_{30}{ }^{2}+\left(3 \eta_{3} \quad-\eta^{2}\right)^{2}\right. \\
& \phi_{4}^{3}=\left(\eta_{30}^{30}+\eta_{12}^{12}+\left(\eta_{11}+\eta_{03}^{2}{ }^{23}\right.\right. \\
& \phi=\left(\eta_{i 0}-3 \eta_{12}\right)\left(\eta_{i: 0}+\eta_{1:}\right)
\end{aligned}
$$

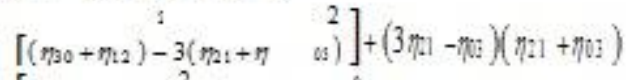

$$
\begin{aligned}
& {\left[3\left(\eta_{30}+3 m_{12}\right)^{2}-\left(\eta_{11}+\eta_{03}\right)\right]^{2}} \\
& \phi=\left(\eta_{00}-\eta_{m}\right)\left(\left[\eta_{n i}-\eta_{n:}\right)^{2}-\left(\eta_{i 1}+\eta_{n i}\right)\right]^{2} \\
& \left.+4 \eta_{11}\left(\eta_{30}+\eta\right)_{12}\right)\left(\eta_{21}+\eta_{03}\right] \\
& \phi_{1}=\left(3 \eta_{21}-\eta_{03}\right)\left(\eta_{30}+\eta_{12}\right)\left(\left[\eta_{30}+\eta_{12}\right)-3\left(\eta_{1}{ }_{2}+\eta_{12}\right)^{2}\right] \\
& +\left(3 m_{12}-m_{00}\right)\left(m_{1}+m_{01}\right)\left[3\left(\eta_{20}+m_{12}\right)-3\left(m_{21}+m_{3}\right)\right]
\end{aligned}
$$

This set of moments is invariant to translation, rotation, and scale change

A total of $7 \times 4=28$ features per face image are computed. These features are stored in the feature database. All the face images in the database are subjected for this feature extraction procedure.

\subsection{Matching}

For a given query image the features are extracted and compared with the other face images by computing distance using Canberra distance measure [9]. The Canberra distance measure is given by: 


\section{ALGORITHM:}

$\operatorname{CanbDist}(x, y)=\sum_{i=1}^{k} \frac{\left|x_{i}-y_{i}\right|}{\left|x_{i}\right|+\left|y_{i}\right|}$

Where $\mathrm{x}$ and $\mathrm{y}$ are the feature vectors of the 2 images being compared and $\mathrm{k}$ is number of features [10] [11]

The distance vector thus obtained is sorted and the top 10 images are considered. The accuracy of matching is calculated by comparing the correct number of matches of the same class as the query class.

\section{EXPERIMENTAL SETUP}

\subsection{Data Set}

The experiments are carried out on ORL database. The ORL database consists of detected face images of 40 individuals. For each of the 40 categories there are 10 samples with different poses expressions

\subsection{Query Set}

The Query set is chosen arbitrarily from the data set against which match is found

\section{RESULTS}

The experiment is carried out shows that out of top 5 images 4 matches are found. An overall of $84 \%$ accuracy was achieved by this method.

\section{CONCLUSION}

In this paper, a novel method for face recognition, using wavelet based features obtained is presented. The experimental results are obtained by choosing the images of different intensity, poses, illuminations and expressions. The Haar wavelet is used, since it is more effective in edge image extraction compared to other wavelets. The features extracted are used as template and moment invariants are used for shape estimation. The experimental results demonstrate the efficacy of the proposed method for face recognition

\section{REFERENCES}

[1] Hua GuGuangda Su Cheng Du, "Feature Points Extraction from Faces", Proceedings of the Imageand Vision Computing, New Zealand, 2003.

[2] S. Y. Lee, Y. K. Ham and R. H. Park, "Recognition of Human Front Faces Using Knowledge-Based Feature extraction and Neuro-Fuzzy Algorithm", Pattern Recognition, 1996, 9(11), pp 1863-1876.

[3] Gaurav Mittal ,SreelaSasi ,“ Robust Preprocessing Algorithm for Face Recognition"Proceedings of the $3^{\text {rd }}$ Canadian conference on Computer and Robot vision, United States ofAmerica, 2006.

[4] R. S. Feris, T. E. de Campos and R.M. Cesar Junior, "Detection and tracking of facial features in video sequences", Lecture Notes in ArtificialIntelligence, 2000, 1793(4), pp. 127-135.

[5] P.S.Hiremath, S. Shivashankar, and JagadeeshPujari "Wavelet based features for color texture classification with application to cbir", IJCSNSInternational Journal of Computer Science and Network Security, VOL.6 No.9A, September 2006
[6] BrunelliRroberto, PoggioTomaso "Face recognition: features versus templates", IEEE transactions on pattern analysis and machine intelligence, vol-15,no2,2007

[7] Omachi Shinichiro, OmachiMasako"fast template matching with polynomials" IEEEtransactions on image processing,vol-16,no-8,2007

[8] SakaliMustafa,Lam kin-man, Yan Hong “A faster converging snake algorithm to locate object boundaries, IEEE transactions on imageprocessing,vol-15,no5,2006

[9] Smeets Dirk, Claes Peter, HermansJeroen , Vandermeulen Dirk, Suetens Paul, "A comparative Study of 3-D Face recognition under expression variations", IEEE transactions on system , man, andCybernetics,vol-42,no-5,2012

[10] Singh Chandan, WaliaEkta, Mittal Neeraj "Fusion of Zernike moments and SIFT features for improved face recognition" Internationalconference on recent advances and future trends in information technology, IJCA, 2012

[11] LajevardiMehndiSevdi ,Hussain. M. Zahir "Zernike moments for facial expression recognition"International conference oncommunication,computer and power, iccep,2009.

[12] Karungaru Stephen , FukumiMinom and Akamatsunono "Face recognition using Genetic algorithm based template matching" Internationalsymposium on communications and information technologics,2004.

[13] Wang Gamg , DuanHui-Chuan " a template extraction approach for image recognition", IEEE -International symposium on information technology in medicine and education, 2012

[14] Lin Sadrina .W Gao Yang, Liu Ray K.J "Template matching for image prediction: A game-theoretical approach", IEEE ICASSP, 2012

[15] HaddadainaJaved, Ahmadi Majid , Faez Karim "An efficient method for recognition of human faces using higher order pseudo Zernike moments invariant",5thIEEE international conference onautomatic face and gesture recognition, 2002

[16] Li Junhua, Teng Li "Feature difference matrix QNNH for facial expressions recognition "IEEE, Chinese control and decision conference, 2008

[17] Fadzil M.H Ahmad , Choom Cheah Lim "Face recognition system based on neural networks and fuzzy logic" IEEE- International conference onneural network,1997

[18] Xia Xing Su, Yuan GuoTui , Hua Chen Tian “ Study an optimal wavelength decomposition level in infrared and visual light image fusion" IEEE,International conference on measuring technologies and electronics automation, 2010. 